\title{
Deletion of bone-marrow-derived receptor for AGEs (RAGE) improves renal function in an experimental mouse model of diabetes
}

\author{
Greg Tesch • Karly C. Sourris • Shaun A. Summers • Domenica McCarthy • \\ Micheal S. Ward • Danielle J. Borg • Linda A. Gallo • Amelia K. Fotheringham • \\ Allison R. Pettit • Felicia Y. T. Yap • Brooke E. Harcourt • Adeline L. Y. Tan • \\ Joshua Y. Kausman • David Nikolic-Paterson • Arthur R. Kitching • Josephine M. Forbes
}

Received: 11 April 2014 / Accepted: 9 May 2014 /Published online: 24 June 2014

(C) Springer-Verlag Berlin Heidelberg 2014

\begin{abstract}
Aims/hypothesis The AGEs and the receptor for AGEs (RAGE) are known contributors to diabetic complications. RAGE also has a physiological role in innate and adaptive immunity and is expressed on immune cells. The aim of this study was to determine whether deletion of RAGE from bonemarrow-derived cells influences the pathogenesis of experimental diabetic nephropathy.

Methods Groups ( $n=8$ /group) of lethally irradiated 8 week old wild-type (WT) mice were reconstituted with bone marrow from WT $(\mathrm{WT} \rightarrow \mathrm{WT}$ ) or RAGE-deficient $(\mathrm{RG})$ mice $(\mathrm{RG} \rightarrow \mathrm{WT})$. Diabetes was induced using multiple low doses of streptozotocin after 8 weeks of bone marrow reconstitution and mice were followed for a further 24 weeks.
\end{abstract}

Electronic supplementary material The online version of this article (doi:10.1007/s00125-014-3291-z) contains peer-reviewed but unedited supplementary material, which is available to authorised users.

G. Tesch $\cdot$ S. A. Summers $\cdot$ D. Nikolic-Paterson $\cdot$ A. R. Kitching Department of Nephrology, Monash Medical Centre,

Monash Health, Clayton, Melbourne, VIC, Australia

G. Tesch • S. A. Summers · J. Y. Kausman • D. Nikolic-Paterson •

A. R. Kitching

Centre for Inflammatory Diseases, Monash University Department

of Medicine, Monash Medical Centre, Clayton, Melbourne, VIC,

Australia

K. C. Sourris • F. Y. T. Yap • B. E. Harcourt • A. L. Y. Tan •

J. M. Forbes

Baker IDI Heart and Diabetes Institute, Melbourne, VIC,

Australia

K. C. Sourris

Department of Medicine, Central and Eastern Clinical School, Alfred

Medical Research and Education Precinct (AMREP),

Monash University, Clayton, Melbourne, VIC, Australia
Results Compared with diabetic WT mice reconstituted with WT bone marrow, diabetic WT mice reconstituted with RG bone marrow had lower urinary albumin excretion and podocyte loss, more normal creatinine clearance and less tubulo-interstitial injury and fibrosis. However, glomerular collagen IV deposition, glomerulosclerosis and cortical levels of TGF- $\beta$ were not different among diabetic mouse groups. The renal tubulo-interstitium of diabetic $\mathrm{RG} \rightarrow$ WT mice also contained fewer infiltrating $\mathrm{CD} 68^{+}$macrophages that were activated. Diabetic $\mathrm{RG} \rightarrow$ WT mice had lower renal cortical concentrations of CC chemokine ligand 2 (CCL2), macrophage inhibitory factor (MIF) and IL-6 than diabetic $\mathrm{WT} \rightarrow \mathrm{WT}$ mice. Renal cortical RAGE ligands S100 calgranulin (S100A)8/9 and AGEs, but not high mobility

D. McCarthy $\cdot$ M. S. Ward • D. J. Borg • L. A. Gallo •

A. K. Fotheringham - B. E. Harcourt - J. M. Forbes $(\bowtie)$

Glycation and Diabetes, Mater Research Institute, University of Queensland, Translational Research Institute, 37 Kent Street,

Woolloongabba, Brisbane, QLD 4010, Australia

e-mail: Josephine.forbes@mater.uq.edu.au

A. R. Pettit

Bones and Immunology Group, Mater Research Institute, University of Queensland, Translational Research Institute, Woolloongabba,

Brisbane, QLD, Australia

J. M. Forbes

Mater Clinical School, University of Queensland, St Lucia, Brisbane, QLD, Australia

J. M. Forbes

Department of Medicine, University of Melbourne, Austin Hospital, Heidelberg, Melbourne, VIC, Australia 
box protein B-1 (HMGB-1) were also decreased in diabetic $\mathrm{RG} \rightarrow \mathrm{WT}$ compared with diabetic WT $\rightarrow$ WT mice. In vitro, bone-marrow-derived macrophages from WT but not RG mice stimulated collagen IV production in cultured proximal tubule cells.

Conclusions/interpretation These studies suggest that RAGE expression on haemopoietically derived immune cells contributes to the functional changes seen in diabetic nephropathy by promoting macrophage infiltration and renal tubulo-interstitial damage.

Keywords Diabetes $\cdot$ Inflammation $\cdot$ Kidney $\cdot$ Macrophages

\begin{tabular}{|c|c|}
\hline \multicolumn{2}{|c|}{ Abbreviations } \\
\hline AMREP & $\begin{array}{l}\text { Alfred Medical Research and Education } \\
\text { Precinct }\end{array}$ \\
\hline CCL2 & $\mathrm{CC}$ chemokine ligand 2 \\
\hline CML & Carboxymethyllysine \\
\hline $\mathrm{CrCl}$ & Creatinine clearance \\
\hline (e)GFP & (enhanced) Green fluorescent protein \\
\hline $\mathrm{GHb}$ & Glycated haemoglobin \\
\hline HMGB-1 & High mobility box protein B-1 \\
\hline $\mathrm{M}-\mathrm{CSF}$ & Macrophage-colony stimulating factor \\
\hline MIF & Macrophage inhibitory factor \\
\hline RAGE & Receptor for AGEs \\
\hline RG & RAGE-deficient \\
\hline $\mathrm{RG} \rightarrow \mathrm{WT}$ & $\begin{array}{l}\text { WT mice reconstituted with bone marrow from } \\
\text { RG mice }\end{array}$ \\
\hline S100A & S100 calgranulin \\
\hline$\alpha$-SMA & $\alpha$ Smooth muscle actin \\
\hline UAER & Urinary AER \\
\hline WT & Wild-type \\
\hline $\mathrm{WT} \rightarrow \mathrm{WT}$ & $\begin{array}{l}\text { WT mice reconstituted with bone marrow from } \\
\text { WT mice }\end{array}$ \\
\hline WT-1 & Wilm's tumour antigen \\
\hline
\end{tabular}

\section{Introduction}

Diabetes and its associated complications, including retinopathy, neuropathy and nephropathy, are an increasing worldwide burden on healthcare systems. Although diabetic nephropathy is the leading cause of end-stage renal disease in developed nations as well as a major risk factor for cardiovascular disease, the pathological mechanisms leading to its development and progression remain to be fully elucidated. It has been well established, however, that both metabolic and haemodynamic factors are involved in the development of diabetic nephropathy in the context of genetic susceptibility [1].

Infiltrating macrophages are a major feature of the kidney inflammatory response in patients with diabetic nephropathy
[2-7], and are thought to become activated through specific receptors. Studies in experimental animal models of diabetes have identified a functional role for both macrophages and the receptor for AGEs (RAGE) in the pathogenesis of diabetic nephropathy [8-11]. RAGE is expressed on macrophages and other circulating and resident tissue cells, and has a physiological role in antigen presentation, dendritic cell homing to lymph nodes and T cell priming [12]. RAGE signal transduction via interaction with ligands such as S100 calgranulin (S100A)8/9, high mobility box protein B-1 (HMGB-1) and AGEs, is thought to induce a proinflammatory response, resulting in the production of chemoattractant molecules, such as CC chemokine ligand 2 (CCL2), and adhesion molecules [13]. More recently, the interaction of RAGE with ligands S100A8/9 has been demonstrated as critical for the proliferation of bone marrow myeloid progenitor cells [14]. The deletion of RAGE from bone marrow cells through chimerism also protects against the development of atherosclerosis in both the presence [14] and the absence [15] of experimental diabetes.

Hence, in this present study, we used bone marrow transplantation from either wild-type (WT) control or RG mice to generate chimeras. We then induced diabetes in these chimeric mice to study the contribution of RAGE in bone-marrowderived cells to the development of experimental diabetic nephropathy.

\section{Methods}

Mouse lines Male mice deficient in Ager (encoding the protein RAGE; RG on C57BL/6J background) and their WT C57BL/6J controls were bred and maintained at the Alfred Medical Research and Education Precinct (AMREP) Animal Services, Melbourne, VIC, Australia. RG mice had been previously backcrossed to $\mathrm{C} 57 \mathrm{BL} / 6 \mathrm{~J}$ for nine generations after generated by insertion of enhanced green fluorescent protein (eGFP) into intron 3 of Ager [16]. The protocols followed for animal handling and experimentation were in accordance with ethical guidelines of the AMREP Animal Ethics Committee, Monash University Animal Ethics Committee and the National Health and Medical Research Council of Australia.

Generation of chimeric mice To generate chimeric mice, groups of recipient male WT (CD45.2 $2^{+}$mice (6 weeks of age; $n=8$ /group) were lethally irradiated with two total body exposures of $5.5 \mathrm{~Gy}, 3 \mathrm{~h}$ apart. Recipient mice were then intravenously injected with $5 \times 10^{6}$ cells from 6 -week-old male donor mice within $24 \mathrm{~h}$ of irradiation. WT (CD45.2 $\left.{ }^{+}\right)$recipient mice were transplanted with bone marrow from either RG donors expressing GFP $(\mathrm{RG} \rightarrow \mathrm{WT}$; expressing eGFP) or WT CD45.1 congenic donors (WT $\rightarrow \mathrm{WT}$; expressing CD45.1). 
Bone marrow reconstitution occurred over 8 weeks and successful chimerism $(94 \pm 1 \%)$ was established by collecting tail vein blood and performing flow cytometry analysis for CD45.1 and eGFP.

Experimental diabetes induction Chimeric mice were rendered diabetic 8 weeks after bone marrow reconstitution via intraperitoneal injection of low-dose streptozotocin (55 mg kg ${ }^{-1}$ day $^{-1}$; MP Biomedicals, Eschwege, Germany) or vehicle (sodium citrate buffer, $\mathrm{pH} 4.5$ ) for 5 consecutive days as previously described $[9,10]$. Throughout the duration of the study, mice were given standard mouse chow and water ad libitum while kept on a $12 \mathrm{~h}$ day/night cycle. After 24 weeks, mice were anaesthetised and exsanguinated by cardiac puncture. Kidneys were removed and were either snap frozen for further analysis or processed for histological analyses.

Measurement of physiological and biochemical variables During week 23 of the study, mice were individually placed into metabolism cages (Tecniplast, Buguggiate, VA, Italy), for a period of $24 \mathrm{~h}$. A blood sample was taken, body weight, food and water intake monitored and urine collected. Glycated haemoglobin $(\mathrm{GHb})$ was measured in whole blood by HPLC (CLC330 GHb Analyser; Primus, Kansas City, MO, USA) as previously described $[9,10]$.

Measurement of renal function Urinary AER (UAER) was determined from $24 \mathrm{~h}$ urine collections by ELISA (Bethyl Laboratories, Montgomery, TX, USA) as per the manufacturer's instructions. Creatinine clearance, corrected for body surface area, was calculated in timed urine and plasma samples following processing with acetyl nitrile and centrifugation at $4{ }^{\circ} \mathrm{C}$. The supernatant fractions were then dried in a Speedivac, resuspended in $10 \mathrm{mmol} / \mathrm{l}$ ammonium acetate, $\mathrm{pH}$ 3.2 and injected into a $\mathrm{C}_{18}$ column (Waters Division, Millipore, Marlborough, MA, USA). HPLC was used for detection of creatinine at $235 \mathrm{~nm}$ using a Hewlett Packard photodiode array (PDA) detector [10].

Histological assessment of kidney injury and leucocyte infiltration Formalin-fixed kidney sections (2 $\mu \mathrm{m})$ were stained with periodic acid-Schiff's reagent and haematoxylin for the assessment of glomerulosclerosis by semi-quantitative scoring of 20 glomerular cross sections, and tubulo-interstitial fibrosis quantified by point counting using a 100 point grid [17]. Immunohistochemistry for Wilm's tumour antigen (WT-1; 1:200; Santa Cruz Biotechnology, Santa Cruz, CA, USA) was performed on $4 \mu \mathrm{m}$ methyl Carnoy's fixed sections. Frozen sections $(5 \mu \mathrm{m})$ from kidneys fixed in periodate lysine paraformaldehyde (PLP) were stained for infiltrating $\mathrm{CD}^{+}, \mathrm{CD}^{+}, \mathrm{CD}^{+} 9^{+}, \mathrm{CD} 11 \mathrm{c}^{+}$or $\mathrm{CD}^{+} 8^{+}$(BD Biosciences, Franklin Lakes, NJ, USA) leucocytes as well as CD169 for activated monocytes/macrophages (1:100; Serotec, Oxford, UK). Cells staining for CD45.1 and CD45.2 were also visualised in frozen sections (1:100, Abcam, Cambridge, UK, or eBioscience, San Diego, CA, USA). Collagen IV (1:20; Millipore, CA, USA) and $\alpha$ smooth muscle actin ( $\alpha$-SMA), for myofibroblast accumulation $(1: 1,000$; Millipore), were localised using immunofluorescence, visualised by confocal microscopy and quantified using Image J software (Rockville, MD, USA).

Renal fractionation In total, $50 \mathrm{mg}$ renal cortex was homogenised (Polytron PT-MR2100, Kinematica, Lucerne, Switzerland) in extraction buffer (20 mmol/1 HEPES buffer, pH 7.2, $1 \mathrm{mmol} / 1$ EGTA, $210 \mathrm{mmol} / 1 \mathrm{mannitol}, 70 \mathrm{mmol} / \mathrm{l}$ sucrose) to isolate cytosol and membranous fractions as previously described $[18,19]$. Total protein was determined by the bicinchoninic acid method (Pierce, Rockford, IL, USA), according to the manufacturer's protocol.

Real-time RT-PCR Total RNA was extracted from kidney cortex using Trizol (Invitrogen) and reverse transcribed with random primers using the Superscript First-Strand Synthesis kit (Invitrogen). Real-time PCR was performed on a StepOne Real-Time PCR System (Applied Biosystems, Scoresby, VIC, Australia) with thermal cycling conditions of $37^{\circ} \mathrm{C}$ for $10 \mathrm{~min}$, $95^{\circ} \mathrm{C}$ for $5 \mathrm{~min}$, followed by 50 cycles of $95^{\circ} \mathrm{C}$ for $15 \mathrm{~s}, 60^{\circ} \mathrm{C}$ for $20 \mathrm{~s}$ and $68^{\circ} \mathrm{C}$ for $20 \mathrm{~s}$. The primer pairs and carboxyfluorescein-labelled minor-groove-binding probe used are described in electronic supplementary material (ESM) Table 1. The relative amount of mRNA was calculated using the comparative $C_{t}\left({ }^{\Delta \Delta} C_{t}\right)$ method. All specific amplicons were normalised against $18 \mathrm{~S}$ rRNA, which was amplified in the same reaction as an internal control using commercial assay reagents (Applied Biosystems). Each of the primer/probe sets were pre-tested and determined to have equivalent PCR amplification efficiencies and are expressed compared with $18 \mathrm{~S}$.

Chemotactic and inflammatory markers Renal cortical IL-6 (R\&D Systems, Minneapolis, MN, USA), CCL2 (RayBiotech, Norcross, GA, USA), macrophage inhibitory factor (MIF; USCN Life Sciences, Hubei, China) and S100A8/9 (Immundiagnostik, Bensheim, Germany) were measured according to the manufacturer's instructions. Serum S100A8/9 (Immundiagnostik) as well as plasma mouse macrophage-colony stimulating factor (M-CSF; R\&D Systems), were assayed by ELISA according to the manufacturer's instructions. Urinary and plasma AGE-modified (carboxymethyllysine [CML]) albumin were assayed via ELISA as previously described [9].

Harvesting of bone-marrow-derived macrophages Bone marrow was isolated aseptically from the femurs of 
8-week-old male donor WT (C57BL/6J) and RG mice. Isolated bone marrow was washed, counted and stained for the expression of CD3 (T cells), CD11b (macrophages/ monocytes), CD19 (B cells) and CD11c (dendritic cells) with pre-conjugated antibodies for flow cytometry (BD Biosciences). A minimum of 10,000 events were acquired on the FACScanto and were analysed by Flowlogic (Inivai, Mentone, VIC, Australia).

For differentiation studies, bone marrow cells were harvested, washed, counted and then resuspended in RPMI containing M-CSF $\left(10^{6} \mathrm{U} / \mathrm{ml}\right)$ and cultured for 5 days. The medium was then collected from control WT- and RGderived bone-marrow macrophages and expression of F4/80 and CD169 on cells was determined using flow cytometry. Bone-marrow-derived macrophages were then incubated for $10 \mathrm{~h}$ in the presence of AGE-BSA $(100 \mu \mathrm{g} / \mathrm{ml})$ or BSA. At the end of $10 \mathrm{~h}$, the conditioned medium from bone-marrowderived macrophages was placed onto cultured proximal tubules cells (PTCs; HK-2 cells) for $6 \mathrm{~h}$ and collagen IV expressed by PTCs was examined by confocal microscopy. Cell culture medium from macrophages exposed to AGE-BSA was also incubated ex vivo with the AGE inhibitor alagebrium chloride for $2 \mathrm{~h}$ pre-exposure to PTCs $(40 \mu \mathrm{mol} / \mathrm{l})$ [17].

Statistical analysis Data are expressed as means \pm SD, unless otherwise stated. Analyses of data were performed using Student's $t$ tests. Data for albuminuria were not normally distributed and were therefore analysed after logarithmic transformation. A $p$ value of less than 0.05 was considered statistically significant.

\section{Results}

Physiological and metabolic variables $\mathrm{GHb}$ and fasting blood glucose concentrations in all chimeric mice were within diabetic ranges following injection of streptozocin (Table 1). $\mathrm{GHb}$ was further increased by week 24 of diabetes (Table 1) compared with week 12 (Table 1), which was consistent in all chimeric groups. Body weights and kidney weight:body weight ratios were not different between diabetic mouse groups (Table 1), but were elevated by diabetes.

Renal function and structure Polyuria was evident by week 24 of diabetes in WT $\rightarrow$ WT mice compared with $\mathrm{RG} \rightarrow \mathrm{WT}$ and RG and WT control mice (Table 1). Consistent with this, diabetic WT $\rightarrow$ WT mice had increased glomerular filtration rates (creatinine clearance [CrCl]; Fig. 1a) and increased urinary albumin excretion over $24 \mathrm{~h}$ at week 12 (Table 1) and week 24 compared with control mice (Fig. 1b); the increases were attenuated in diabetic $\mathrm{RG} \rightarrow \mathrm{WT}$ mice (Table 1; Fig. 1a, b). At week 24, $\mathrm{CrCl}$ was decreased in $\mathrm{RG}$ compared with WT mice (Fig. 1a). There was also increased expansion of the tubulo-interstitium seen with diabetes in WT $\rightarrow$ WT mice, which was significantly less in diabetic $\mathrm{RG} \rightarrow \mathrm{WT}$ mice (Fig. 1c, g, h). Glomerulosclerosis was not different between diabetic mouse groups but was increased by diabetes (Fig. 1d). The renal cortical expression of mouse Tim-1 gene (also known as Havcrl) encoding kidney injury molecule (KIM-1) was elevated by diabetes in WT $\rightarrow$ WT mice but to a lesser degree in diabetic RG $\rightarrow$ WT mice (Fig. 1e). WT- $1^{+}$ cells within a glomerular cross section were also decreased with diabetes in WT $\rightarrow$ WT mice, and this was attenuated in
Table 1 Physiological and biochemical variables in nondiabetic control and mouse chimeras at week 3,12 and 24 of diabetes
${ }^{*} p<0.05$ vs WT $\rightarrow \mathrm{WT} ;{ }^{\dagger} p<0.05$

vs WT $\rightarrow$ WT at week 12 ;

${ }^{\ddagger} p<0.05$ vs $\mathrm{RG} \rightarrow \mathrm{WT}$ at week 12 ;

${ }_{p}^{\$_{p}}<0.05$ vs RG $\rightarrow$ WT at week 24

BW, body weight; FBG, fasting blood glucose; KW, kidney weight

\begin{tabular}{|c|c|c|c|c|}
\hline \multirow[t]{2}{*}{ Variable } & \multicolumn{4}{|l|}{ Mouse } \\
\hline & WT $(n=10)$ & $\mathrm{RG}(n=10)$ & $\mathrm{WT} \rightarrow \mathrm{WT}(n=8)$ & $\mathrm{RG} \rightarrow \mathrm{WT}(n=7)$ \\
\hline \multicolumn{5}{|l|}{ Week 3} \\
\hline FBG $(\mathrm{mmol} / \mathrm{l})$ & ND & ND & $20.8 \pm 7.3$ & $21.5 \pm 7.4$ \\
\hline BW (g) & ND & ND & $23.3 \pm 2.0$ & $21.7 \pm 1.6$ \\
\hline \multicolumn{5}{|l|}{ Week 12} \\
\hline FBG $(\mathrm{mmol} / \mathrm{l})$ & $8.8 \pm 0.4^{*}$ & $9.5 \pm 0.4^{\ddagger}$ & $21.7 \pm 2.0$ & $31.5 \pm 9.4$ \\
\hline BW (g) & $34.4 \pm 1.2^{*}$ & $34.4 \pm 3.4^{\ddagger}$ & $22.6 \pm 1.7$ & $22.1 \pm 0.8$ \\
\hline $\mathrm{GHb}(\%)$ & $3.7 \pm 0.3^{*}$ & $3.5 \pm 0.3^{\ddagger}$ & $7.8 \pm 1.1$ & $7.9 \pm 1.0$ \\
\hline UAER ( $\mu \mathrm{g} /$ day) & $71 \pm 35^{*}$ & $75 \pm 47^{\star}$ & $2,802 \pm 190$ & $300 \pm 119^{*}$ \\
\hline \multicolumn{5}{|l|}{ Week 24} \\
\hline FBG & $8.9 \pm 0.5^{*}$ & $10.1 \pm 1.6^{\S}$ & $33.1 \pm 2.5^{\dagger}$ & $28.8 \pm 2.6$ \\
\hline BW (g) & $35.7 \pm 1.0^{*}$ & $27.0 \pm 2.7^{\S}$ & $21.7 \pm 1.5$ & $22.5 \pm 1.9$ \\
\hline $\mathrm{GHb}(\%)$ & $3.6 \pm 0.2^{*}$ & $4.7 \pm 1.1^{\S}$ & $9.0 \pm 0.6^{\dagger}$ & $9.2 \pm 0.9^{\ddagger}$ \\
\hline Total KW (g) & $0.36 \pm 0.02$ & $0.42 \pm 0.05^{\S}$ & $0.35 \pm 0.04$ & $0.35 \pm 0.05$ \\
\hline $\mathrm{KW}: \mathrm{BW}\left(\times 10^{-3}\right)$ & $1.0 \pm 0.1^{*}$ & $1.1 \pm 0.1^{\S}$ & $1.7 \pm 0.2$ & $1.6 \pm 0.2$ \\
\hline Urine (ml/day) & $1.8 \pm 0.3^{*}$ & $0.6 \pm 0.4^{\S}$ & $12.1 \pm 9.8$ & $2.9 \pm 0.7^{*}$ \\
\hline
\end{tabular}


a

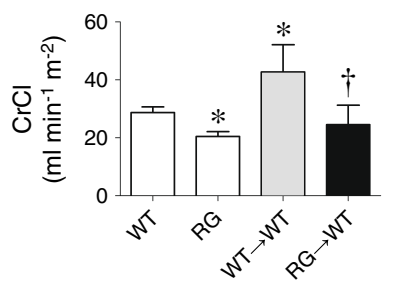

b
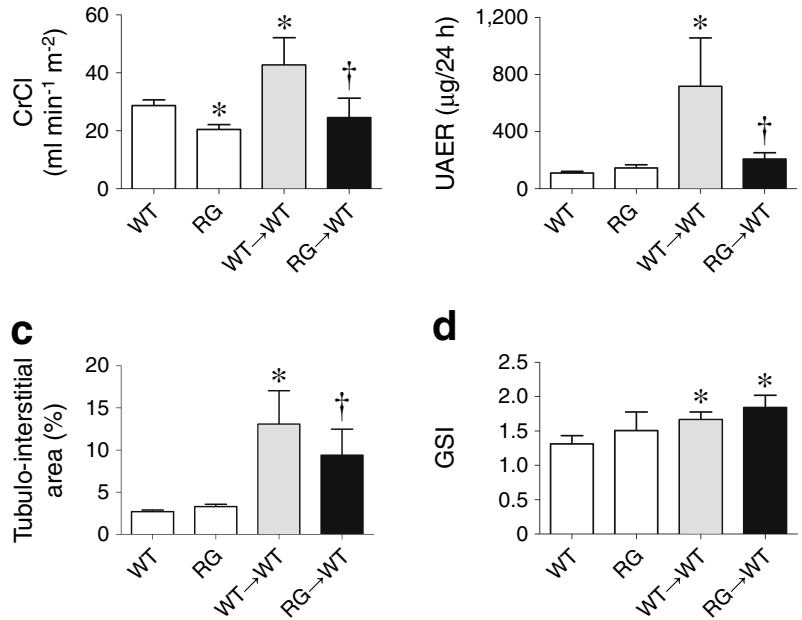

d

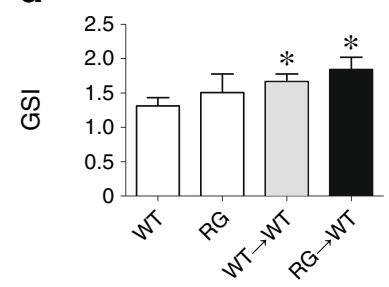

f
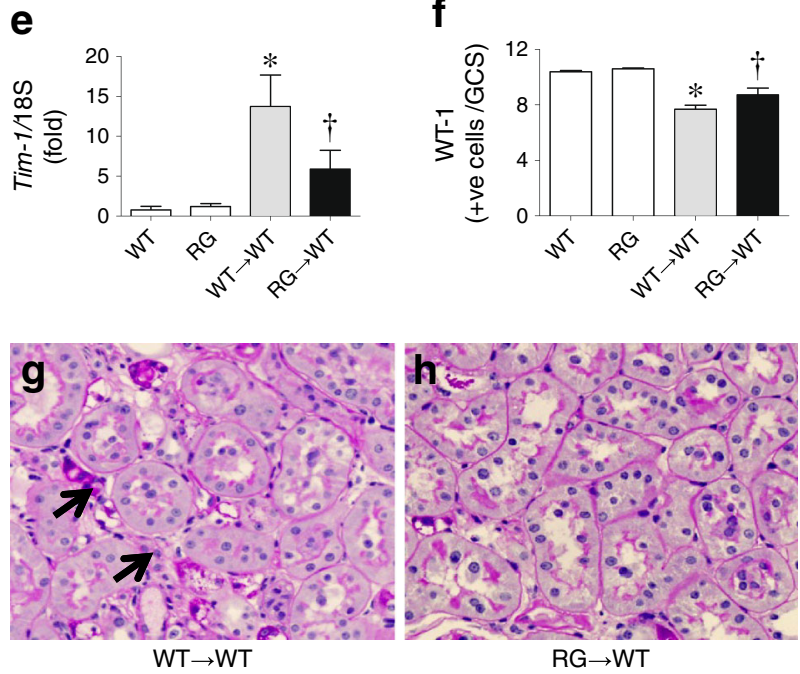

Fig. 1 Renal functional and structural variables at week 24 of diabetes: (a) $\mathrm{CrCl}$ by HPLC, corrected for body surface area; (b) $24 \mathrm{~h}$ UAER; (c) tubulo-interstitial area by morphometric quantification as percentage of total cortical area; (d) glomerulosclerosis; (e) renal cortical gene expression of Tim-1 (encoding kidney injury molecule); and (f) WT-1 in podocytes. (g, h) Photomicrographs of periodic acid-Schiff's reagent staining of renal cortex $(\times 400)$ : $(\mathbf{g})$ diabetic WT $\rightarrow$ WT; and $(\mathbf{h})$ diabetic RG $\rightarrow$ WT. Arrows highlight areas of tubulo-interstitial accumulation. White bars, non-diabetic control; grey bars, diabetic WT $\rightarrow$ WT; black bars, diabetic RG $\rightarrow$ WT. ${ }^{*} p<0.05$ vs WT; ${ }^{\dagger} p<0.05$ vs WT $\rightarrow$ WT. GCS, glomerular cross section; GSI, glomerulosclerosis

diabetic $R G \rightarrow$ WT mice (Fig. 1f). There was no change in renal cortical activation of the profibrotic cytokine TGF- $\beta$ in diabetic $\mathrm{RG} \rightarrow \mathrm{WT}$ when compared with $\mathrm{WT} \rightarrow \mathrm{WT}$ mice $(\mathrm{WT} \rightarrow \mathrm{WT}, 43.9 \pm 27.7 \mathrm{pg} / \mathrm{ml} ; \mathrm{RG} \rightarrow \mathrm{WT}, 85.4 \pm 83.3 \mathrm{pg} / \mathrm{ml}$ vs WT, $30.9 \pm 5.7 \mathrm{pg} / \mathrm{ml}$; RG, $26.6 \pm 10.1 \mathrm{pg} / \mathrm{ml}$ ).

Collagen IV was increased in the tubulo-interstitium of diabetic WT $\rightarrow$ WT mice compared with WT and RG mice, but this increase was attenuated in kidney cortices from $\mathrm{RG} \rightarrow \mathrm{WT}$ chimeras (Fig. 2a-c). However, there was no significant decrease in glomerular collagen IV in diabetic $\mathrm{RG} \rightarrow \mathrm{WT}$ mice $(\mathrm{WT} \rightarrow \mathrm{WT}, \quad 10.4 \pm 2.5 \%$; $\mathrm{RG} \rightarrow \mathrm{WT}, 8.9 \pm 5.7 \%$ glomerular tuft area).

Cortical $\alpha$-SMA was not increased with diabetes $(p=0.065$, Fig. $2 \mathrm{~d}-\mathrm{f}$ ) nor was there a significant difference seen between diabetic groups $(p=0.075)$.

Circulating and renal markers of inflammation Renal cortices from diabetic WT $\rightarrow$ WT mice had a greater number of interstitial $\mathrm{CD} 68^{+}$macrophages compared with non-diabetic and diabetic RG $\rightarrow$ WT mice (Fig. 3a, h, i). There were also more $\mathrm{CD} 169^{+} \mathrm{CD} 68^{+}$macrophages in the tubulo-interstitium with diabetes in WT $\rightarrow$ WT mice, with lower levels in non-diabetic mice as well as diabetic RG $\rightarrow$ WT mice (Fig. 3b-d). However, there was a modest increase in glomerular macrophages in mice that received RG bone marrow compared with bone marrow from WT donors (Fig. 3e). There were no significant differences among groups for other immune cell types as assessed by immunohistochemistry (Table 2). Renal cortical gene expression of $C d 68$ relative to $18 \mathrm{~S}$ (Fig. 3f) and the M2 marker Fizz-1 (also known as Retnlb) to Cd68 expression (Fig. 3g) were significantly elevated by diabetes in $\mathrm{WT} \rightarrow$ WT but lower in diabetic RG $\rightarrow$ WT mice. There were no changes seen in the gene expression of other M2 markers (arginase, iNos [also known as Nos2]) between diabetic mice (data not shown).

Infiltration of bone-marrow-derived cells into the diabetic kidney of WT $\rightarrow$ WT mice was tracked using immunofluorescence for CD45.1, which showed significant infiltration of cells into both glomeruli and the tubular compartments (Fig. 3j, k). Given that nuclei from Ager-deficient mice contained an eGFP insert, RG bone-marrow-derived cells were also tracked into the kidneys during diabetes. Within diabetic RG $\rightarrow$ WT (Fig. 31, m) $\mathrm{eGFP}^{+}$cells were also present both within the glomeruli and tubulo-interstitium.

Diabetic WT $\rightarrow$ WT had higher levels of several chemokines and cytokines within renal cortices compared with control mice. These included CCL2 (Fig. 4a), MIF (Fig. 4b) and IL-6 protein (Fig. 4c), levels of which were lower in $\mathrm{RG} \rightarrow$ WT diabetic mice. Renal cortical concentrations of the RAGE ligands S100A8/9 (Fig. 4d) and AGEs (Fig. 4e) were increased in diabetic WT $\rightarrow$ WT mice, but attenuated in the diabetic $\mathrm{RG} \rightarrow \mathrm{WT}$ group. By contrast, renal cortical concentrations of the RAGE ligand HMGB-1 (Fig. 4f) were lower in all diabetic mice compared with controls. Plasma M-CSF concentrations were similar in both diabetic mouse groups (WT $\rightarrow \mathrm{WT}, 614 \pm 51 \mathrm{pg} / \mathrm{ml}$; $\mathrm{RG} \rightarrow \mathrm{WT}, 649 \pm 86 \mathrm{pg} / \mathrm{ml}$ ). Circulating concentrations of HMGB-1 and AGEs were decreased by diabetes and there was no difference in circulating S100 A8/9 between $\mathrm{WT} \rightarrow \mathrm{WT}$ and $\mathrm{RG} \rightarrow \mathrm{WT}$ mice (data not shown).

In bone marrow from WT mice, there was a greater number of $\mathrm{F} 4 / 80^{+}$macrophages differentiated in the presence of M-CSF (Fig. 5a; 65.5\%) when compared with $\mathrm{Ager}^{-/}$mice 


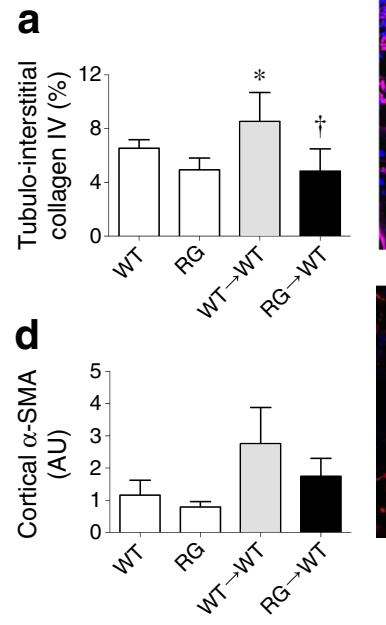

Fig. 2 Morphometric analyses of confocal images $(\times 630)$ of immunofluorescence for $(\mathbf{a}-\mathbf{c})$ tubulo-interstitial collagen IV: (a) quantification as percentage of total cortical area; (b) diabetic WT $\rightarrow$ WT; (c) diabetic $\mathrm{RG} \rightarrow$ WT. (d-f) Similar analyses for $\alpha$-SMA: (d) quantification; (e) diabetic WT $\rightarrow$ WT; (f) diabetic $\mathrm{RG} \rightarrow \mathrm{WT}$. White bars, non-diabetic
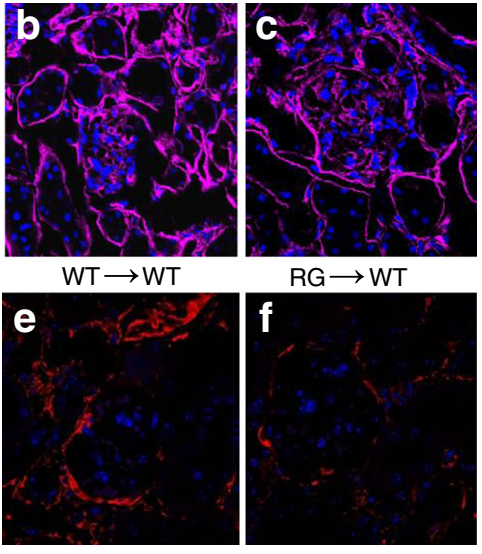

$\mathrm{RG} \rightarrow \mathrm{W}$

control mice; grey bars, diabetic WT recipients reconstituted with WT bone marrow; black bars, diabetic WT mice reconstituted with RG bone marrow. In (a) ${ }^{*} p<0.05$ vs WT; ${ }^{\dagger} p<0.05$ vs WT $\rightarrow$ WT; in (b), $p=0.065$ for WT vs WT $\rightarrow \mathrm{WT}$, and $p=0.075$ for $\mathrm{WT} \rightarrow \mathrm{WT}$ vs $\mathrm{RG} \rightarrow \mathrm{WT}$
Fig. 3 Macrophage accumulation within renal cortices. Control and diabetic chimeric mice were analysed at week 24. (a) Tubulo-interstitial accumulation of $\mathrm{CD} 68^{+}$cells. (b-d) Immunofluorescence for CD169: (b) quantification of tubulo-interstitial $\mathrm{CD} 169^{+}$cells; (c) $\mathrm{CD} 169^{+}$cells in $\mathrm{WT} \rightarrow \mathrm{WT}$; and (d) $\mathrm{CD} 169^{+}$in $\mathrm{RG} \rightarrow \mathrm{WT}$. (e) Glomerular $\mathrm{CD} 68^{+}$cells per glomerular cross section. (f) Renal cortical Cd68 gene expression relative to $18 \mathrm{~S}$ expression. (g) Renal cortical Fizz-1/Cd68 expression. (h-m) Photomicrographs of CD68 immunohistochemistry as well as CD-45.1 and eGFP immunofluorescence in renal cortical sections: (h) CD68 in $\mathrm{WT} \rightarrow \mathrm{WT}(\times 200)$; (i) CD68 in $\mathrm{RG} \rightarrow \mathrm{WT}(\times 200)$.

Photomicrographs of infiltrating bone-marrow-derived cells labelled with CD45.1 (red, WT; magenta, CD45.2) at: (j) $\times 630$; (k) $\times 200$; and eGFP fluorescence at $(\mathbf{l}) \times 630$; and $(\mathbf{m}) \times 200(\mathrm{RG}$, green) within renal cortices at week 24 of diabetes. Arrows indicate bone-marrow-derived cells. White bars, non-diabetic control; grey bars, diabetic $\mathrm{WT} \rightarrow \mathrm{WT}$; black bars, diabetic $\mathrm{RG} \rightarrow \mathrm{WT} .{ }^{*} p<0.05$ vs WT; ${ }^{\dagger} p<0.05$ vs WT $\rightarrow$ WT. GCS, glomerular cross section

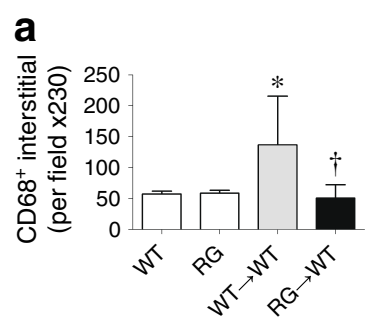

\section{b}

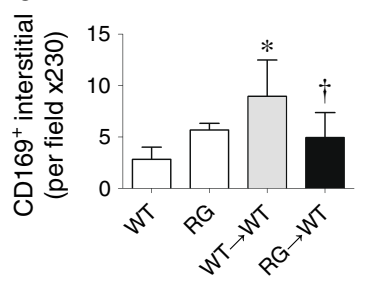

f
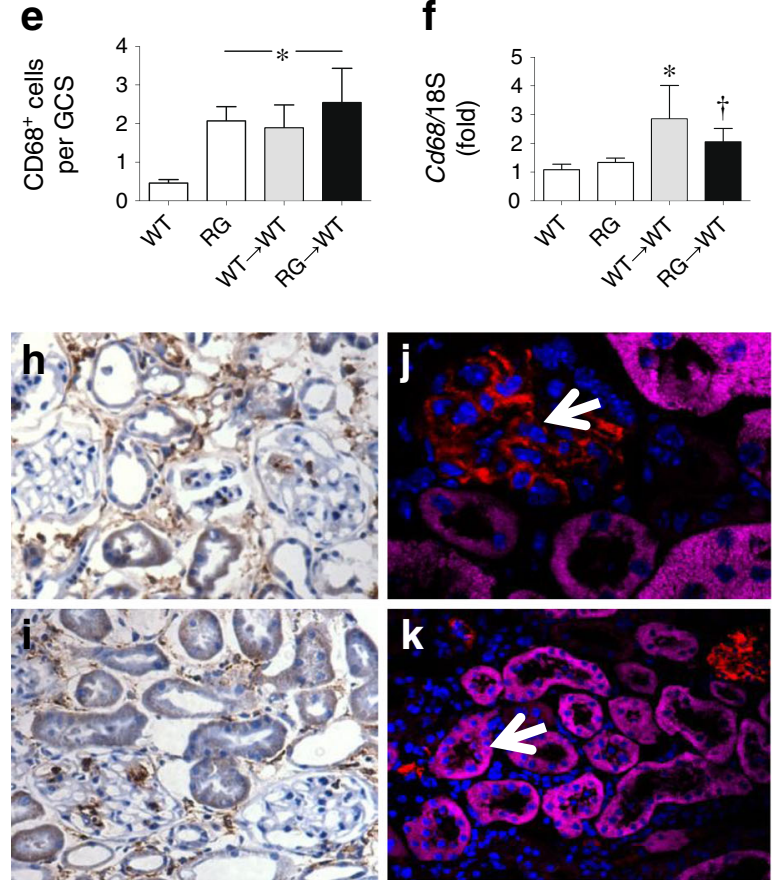
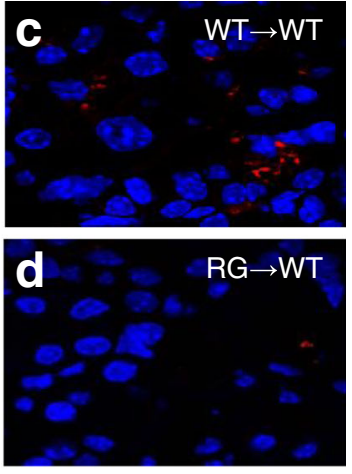

g
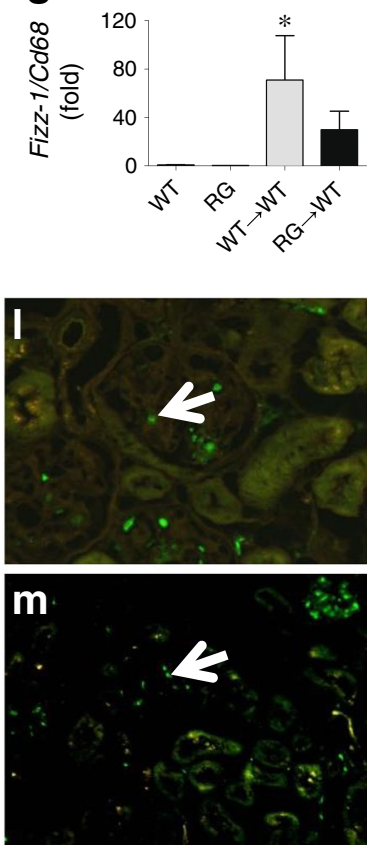
Table 2 Morphometric quantification of renal cortical lymphocytes in chimeric mice at week 24 of diabetes

\begin{tabular}{lll}
\hline Marker & $\mathrm{WT} \rightarrow \mathrm{WT}(n=8)$ & $\mathrm{RG} \rightarrow \mathrm{WT}(n=7)$ \\
\hline $\mathrm{CD} 4$ & $1.07 \pm 0.56$ & $0.63 \pm 0.29$ \\
$\mathrm{CD} 8$ & $0.16 \pm 0.17$ & $0.22 \pm 0.23$ \\
$\mathrm{CD} 11 \mathrm{c}$ & $2.73 \pm 1.04$ & $1.80 \pm 0.47$ \\
$\mathrm{CD} 19$ & $0.51 \pm 0.35$ & $0.48 \pm 0.24$ \\
\hline
\end{tabular}

Data are expressed as cells/field $(\times 100)$

(Fig. 5b; 47.2\%). Bone marrow derived from $\mathrm{Ager}^{-/}$mice had fewer $\mathrm{CD} 11 \mathrm{~b}^{+}$cells (Fig. 5c), and increased numbers of $\mathrm{CD}_{11 \mathrm{c}^{+}}$cells, but there was no difference between the total cell numbers harvested from the bone marrow (WT $2.34 \times 10^{7}$ cells vs RG $2.28 \times 10^{7}$ ). A greater number of WT bonemarrow-differentiated macrophages were also F4/ $80^{+} \mathrm{CD} 169^{+}$following incubation with AGE-BSA $(25.6 \%)$ compared with BSA (Fig. 5d, 19.3\%). PTCs incubated with conditioned media collected from WT bone-marrow-derived macrophages exposed to AGE-albumin showed an increased expression of collagen IV (Fig. 5e) compared with conditioned media from macrophages exposed to unmodified albumin (Fig. 5f) or to AGE-albumin pre-incubated with alagebrium chloride (Fig. 5g). PTCs incubated with conditioned media collected from Ager $^{-/}$bone-marrow-derived macrophages exposed to AGE-albumin (Fig. 5h) also had a reduced expression of collagen IV when compared with conditioned media obtained from AGE-albumin-exposed WT bone-marrow-derived macrophages.

\section{Discussion}

Within the present study, reconstitution using RG bone marrow $(\mathrm{RG} \rightarrow \mathrm{WT})$ attenuated the development and progression of kidney disease when mice were rendered diabetic. Indeed, decreases in albuminuria were even evident at a relatively early time point (after 12 weeks of diabetes), as well as by the study endpoint at week 24 , with concomitant retention of podocytes as well as less tubulo-interstitial injury and macrophage infiltration and activation. This protection was associated with better renal function in $\mathrm{RG} \rightarrow \mathrm{WT}$ diabetic mice. We also demonstrated that bone-marrow-derived macrophages from WT mice exposed to AGEs can induce greater collagen IV expression in PTCs in vitro, which was not seen in macrophages from Ager-knockout mice. Taken together, these findings suggest that one of the major defects seen as a result of increases in RAGE expression on infiltrating immune cells into the diabetic kidney is tubulo-interstitial fibrosis, coupled to changes in renal function.

Given that the transplanted bone marrow from RG mice expressed GFP and bone marrow from WT mice expressed CD45.1, we followed reconstitution and renal infiltration during diabetes. The images suggested that bone-marrow-derived cells derived from both donor types had migrated to recipient kidneys. There was no evidence that bone-marrow-derived cells with RAGE deficiency do not undergo monocytosis/ myelopoiesis and migrate into the kidney tubulo-interstitium as suggested previously [4, 5, 20-22]. There were, however, lower concentrations of the chemotactic molecule CCL2 in kidneys from these mice. Further, a number of infiltrating cells in diabetic mouse renal cortices from mice reconstituted with WT bone marrow were $\mathrm{CD} 68^{+}$monocytes/macrophages or cells that had migrated to glomeruli, where there was no difference in macrophage number between diabetic groups. Indeed, there were no significant changes in other infiltrating immune cell types identified between diabetic mouse groups in the present study. This is consistent with a previous report from our group [23] suggesting that macrophages are the major immune cells contributing to renal functional changes and tubulo-interstitial fibrosis in experimental diabetes. There
Fig. 4 Cytokines, chemokines and RAGE ligands. Renal cortices taken from diabetic mice were analysed by ELISA at week 24 for protein expression of: (a) CCL2; (b) MIF; (c) IL-6; (d) S100A8/9; (e) AGEs (CML-albumin); and (f) HMGB-1. Black bars, diabetic WT recipients; grey bars, diabetic RG recipients. ${ }^{*} p<0.05$ vs WT; ${ }^{\dagger} p<0.05$ vs WT $\rightarrow$ WT. ND, not detected a
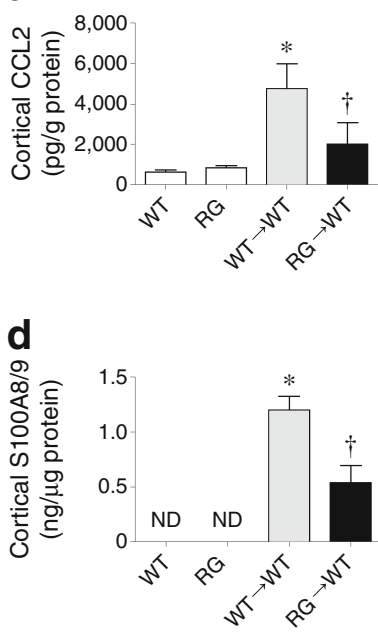

b

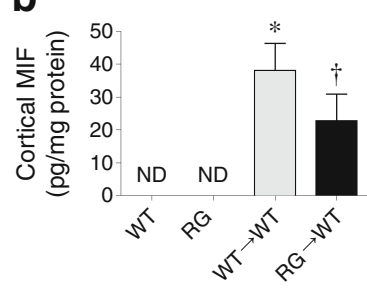

e

C

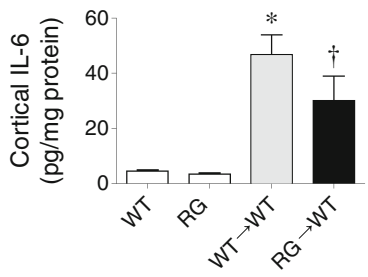

f

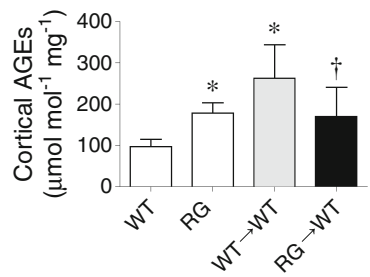

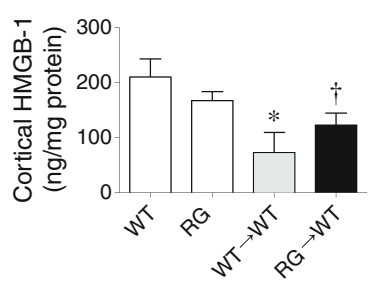


a

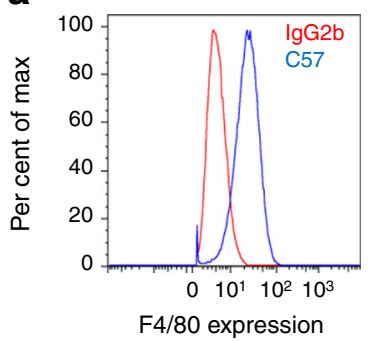

b

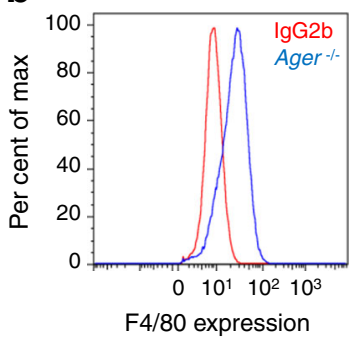

C

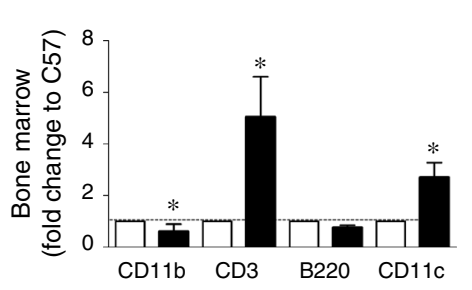

d
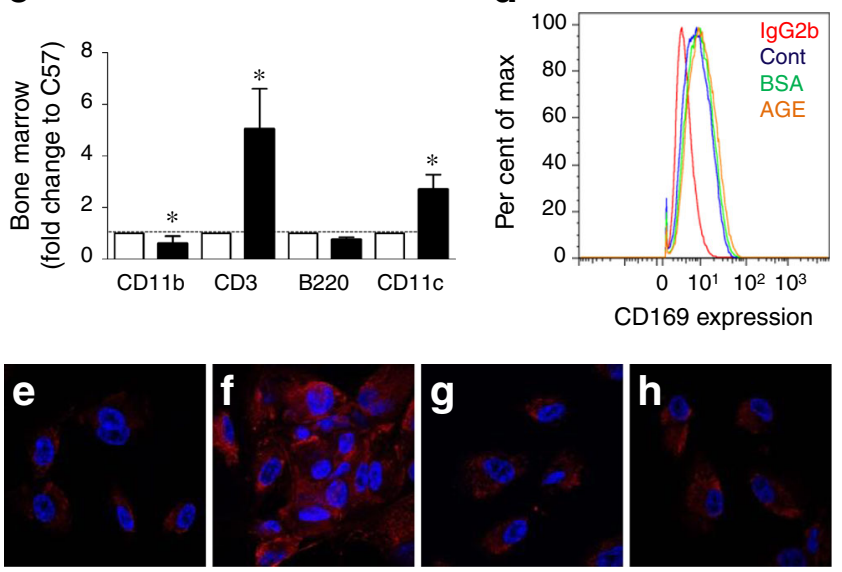

Fig. 5 (a, b) $\mathrm{F} 4 / 80^{+}$bone-marrow-derived macrophages after differentiation for 5 days with M-CSF. (a) WT mice, $65.5 \% \mathrm{~F} 4 / 80^{+}$; (b) $\mathrm{Ager}^{-/}$, $47.2 \% \mathrm{~F} 4 / 80^{+}$. (c) Flow cytometry characterisation of WT and $\mathrm{Ager}^{-{ }^{\prime}}$ bone marrow at transplantation. Data are expressed as fold change above WT (C57BL/6 J). (d) CD169 ${ }^{+} \mathrm{F} 4 / 80^{+}$bone-marrow-derived macrophages following incubation with AGE-albumin (AGE) or unmodified albumin $(\mathrm{BSA} ; 100 \mu \mathrm{g} / \mathrm{ml})$ for $10 \mathrm{~h}$. $(\mathbf{e}-\mathbf{h})$ Confocal images $(\times 630)$ of collagen IV immunofluorescence of HK-2 PTCs after $6 \mathrm{~h}$ exposure to conditioned media harvested from bone-marrow-derived macrophages from: (e) WT incubated with AGE-modified albumin (100 $\mu \mathrm{g} / \mathrm{ml})$; (f) WT mice incubated with unmodified albumin $(100 \mu \mathrm{g} / \mathrm{ml})$; (g) WT incubated with AGE-modified albumin and AGE-lowering pharmacotherapy alagebrium chloride $(40 \mu \mathrm{mol} / \mathrm{l})$ pre-exposure; (h) $\mathrm{Ager}^{-/}$mice incubated with AGE-modified albumin. B220, B cells; CD3, T cells; CD11b, monocytes/macrophages; CD11c, dendritic cells. White bars, WT; black bars, RAGE-deficient bone marrow cells. * ${ }^{*}<0.05$ vs WT by Student's $t$ test. Cont, control; max, maximum

was evidence of reconstitution of glomerular cells by bone marrow from both donor groups as has been previously shown in diabetes [24], but the relative contribution of these remains to be delineated in future studies.

Global RAGE deficiency has been shown to provide protection against renal injury in experimental models of diabetes $[9,10]$. However, these studies were not able to determine if the deletion of RAGE on infiltrating cells per se was responsible for the improved renal function and structure as identified in the present study. In another study, bone-marrowderived macrophages taken from RG mice produced lower levels of cytokines such as IL-6 [25] on stimulation, which implies that RAGE may be required for macrophage activation and cytokine production. Further, the renal parenchyma from diabetic mice reconstituted with RG bone marrow contained fewer activated macrophages and lower concentrations of various cytokines. This is further supported by another experimental murine study, where deletion of RAGE from bone marrow mediated immune cell infiltration into atherosclerotic lesions in a non-diabetic context [15]. In addition, we have shown that $\mathrm{F} 4 / 80^{+}$cells derived from $\mathrm{RG}$ bone marrow stimulated less collagen IV production from human PTCs following stimulation with the ligand AGE-BSA, which links the improvements seen in the tubulo-interstitium to the presence of RAGE on the interstitial infiltrate. As tubulointerstitial injury is the most accurate determinant of progressive loss of renal function in chronic kidney disease, including diabetic nephropathy in humans, it is likely that the deletion of RAGE from infiltrating cells contributed directly to the changes in renal function that were observed. It is also plausible that the reduction in RAGE ligand formation that occurred as a result of less renal parenchymal damage in $\mathrm{RG} \rightarrow$ WT diabetic mice also prevented damage to PTCs via RAGE expressed on intrinsic renal cells. Not surprisingly, a number of RAGE ligands are known pathological mediators of proximal tubular damage in diabetic nephropathy [1] and blockade of ligands such as AGEs is traditionally associated with structural and functional improvements in both experimental [17] and human diabetic nephropathy [26].

Overall, this group of studies suggests that changes in RAGE in interstitial infiltrates may be an important modulator of tubulo-interstitial disease and likely podocyte injury in the diabetic kidney. This concept is also supported by a previous study showing that functional blockade of macrophage infiltration in established diabetic nephropathy results in improved renal function, with reductions in interstitial inflammation and fibrosis [27]. Furthermore, deletion of RAGE in bone marrow had significant beneficial effects on the levels of other RAGE ligands, which are pathologically increased in the diabetic kidney, alleviating damage by preventing ligation to RAGE on intrinsic renal cells such as podocytes and PTCs. Hence, targeted dampening of the expression of RAGE on bonemarrow-derived cells appears renoprotective and therefore manipulation of this pathway warrants further investigation in diabetic nephropathy and likely other complications where interstitial infiltration by monocytes and macrophages is a hallmark.

Acknowledgements Our esteemed colleague Dr Shaun Summers, who constructed the bone marrow chimeras used in this research, passed away on 21 September 2013 before publication of this work.

The authors acknowledge M. Arnstein and M. Thomas at Baker IDI Heart and Diabetes Research Institute, Melbourne, VIC, Australia, for technical assistance in histology and HPLC for creatinine clearance, respectively.

Funding JMF is supported by a Senior Research Fellowship from the National Health and Medical Research Council of Australia (NHMRC). LAG and MSW are supported by research fellowships from the Heart Foundation Australia and JDRF International, respectively. FYTY and 
AKF were supported by $\mathrm{PhD}$ scholarships from NHMRC and the University of Queensland, QLD, Australia. This research was supported by the NHMRC and JDRF International. The funding bodies (NHMRC and JDRF) had no role in the study design, data collection and analysis, decision to publish or preparation of the manuscript.

Duality of interest The authors declare that there is no duality of interest associated with this manuscript.

Contribution statement JMF, GT, ARK and DNP each contributed to the conception and design of the study, overseeing the completion of the experiments as well as the drafting of the manuscript. SAS and JYK created the chimeras, assisted with data analyses and drafting of manuscript. FYTY, KCS, ALYT completed the animal studies and assisted with manuscript drafting. BEH performed renal functional measures, interpreted data and assisted with manuscript drafting. DM and DJB conceived, completed the histology and confocal microscopy as well as manuscript and figure preparation. LAG, ARP and MSW conceived, completed the macrophage in vitro experiments, flow cytometry, analysed the data and assisted with manuscript critical revision. AKF completed the ELISAs, analysed and interpreted data and critically revised the manuscript. All of the authors have approved the final version. JMF is the guarantor of this work.

\section{References}

1. Forbes JM, Cooper ME (2013) Mechanisms of diabetic complications. Physiol Rev 93:137-188

2. US Renal Data System (2013) USRDS 2008 annual data report: atlas of chronic kidney disease and end-stage renal disease in the United States. National Institutes of Health, National Institute of Diabetes and Digestive and Kidney Diseases, Bethesda

3. (2010) Association of estimated glomerular filtration rate and albuminuria with all-cause and cardiovascular mortality in general population cohorts: a collaborative meta-analysis. Lancet 375:2073-2081

4. Chow FY, Nikolic-Paterson DJ, Ma FY, Ozols E, Rollins BJ, Tesch GH (2007) Monocyte chemoattractant protein-1-induced tissue inflammation is critical for the development of renal injury but not type 2 diabetes in obese $\mathrm{db} / \mathrm{db}$ mice. Diabetologia 50:471-480

5. Chow FY, Nikolic-Paterson DJ, Ozols E, Atkins RC, Rollin BJ, Tesch GH (2006) Monocyte chemoattractant protein-1 promotes the development of diabetic renal injury in streptozotocin-treated mice. Kidney Int 69:73-80

6. Navarro JF, Mora C, Gomez M, Muros M, Lopez-Aguilar C, Garcia J (2008) Influence of renal involvement on peripheral blood mononuclear cell expression behaviour of tumour necrosis factor-alpha and interleukin-6 in type 2 diabetic patients. Nephrol Dial Transplant 23: 919-926

7. Navarro-Gonzalez JF, Mora-Fernandez C (2008) The role of inflammatory cytokines in diabetic nephropathy. J Am Soc Nephrol 19: 433-442

8. Inagi R, Yamamoto Y, Nangaku M et al (2006) A severe diabetic nephropathy model with early development of nodule-like lesions induced by megsin overexpression in RAGE/iNOS transgenic mice. Diabetes 55:356-366

9. Sourris KC, Morley AL, Koitka A et al (2010) Receptor for AGEs (RAGE) blockade may exert its renoprotective effects in patients with diabetic nephropathy via induction of the angiotensin II type 2 (AT2) receptor. Diabetologia 53:2442-2451

10. Tan AL, Sourris KC, Harcourt BE et al (2010) Disparate effects on renal and oxidative parameters following RAGE deletion,
AGE accumulation inhibition, or dietary AGE control in experimental diabetic nephropathy. Am J Physiol Renal Physiol 298: F763-F770

11. Wendt TM, Tanji N, Guo J et al (2003) RAGE drives the development of glomerulosclerosis and implicates podocyte activation in the pathogenesis of diabetic nephropathy. Am J Pathol 162:1123-1137

12. Bierhaus A, Humpert PM, Morcos M et al (2005) Understanding RAGE, the receptor for advanced glycation end products. J Mol Med (Berl) 83:876-886

13. Bierhaus A, Schiekofer S, Schwaninger M et al (2001) Diabetesassociated sustained activation of the transcription factor nuclear factor-kB. Diabetes 50:2792-2808

14. Nagareddy PR, Murphy AJ, Stirzaker RA et al (2013) Hyperglycemia promotes myelopoiesis and impairs the resolution of atherosclerosis. Cell Metab 17:695-708

15. Morris-Rosenfeld S, Blessing E, Preusch MR et al (2011) Deletion of bone marrow-derived receptor for advanced glycation end products inhibits atherosclerotic plaque progression. Eur J Clin Invest 41: $1164-1171$

16. Liliensiek B, Weigand MA, Bierhaus A et al (2004) Receptor for advanced glycation end products (RAGE) regulates sepsis but not the adaptive immune response. J Clin Invest 113: $1641-1650$

17. Forbes JM, Thallas V, Thomas MC et al (2003) The breakdown of preexisting advanced glycation end products is associated with reduced renal fibrosis in experimental diabetes. FASEB J 17:17621764

18. Coughlan MT, Thallas-Bonke V, Pete J et al (2007) Combination therapy with the advanced glycation end product cross-link breaker, alagebrium, and angiotensin converting enzyme inhibitors in diabetes: synergy or redundancy? Endocrinology 148: $886-895$

19. Thallas-Bonke V, Thorpe SR, Coughlan MT et al (2008) Inhibition of NADPH oxidase prevents advanced glycation end product-mediated damage in diabetic nephropathy through a protein kinase C-alphadependent pathway. Diabetes 57:460-469

20. Chow F, Ozols E, Nikolic-Paterson DJ, Atkins RC, Tesch GH (2004) Macrophages in mouse type 2 diabetic nephropathy: correlation with diabetic state and progressive renal injury. Kidney Int 65:116-128

21. Chow FY, Nikolic-Paterson DJ, Ozols E, Atkins RC, Tesch GH (2005) Intercellular adhesion molecule-1 deficiency is protective against nephropathy in type 2 diabetic $\mathrm{db} / \mathrm{db}$ mice. J Am Soc Nephrol 16: $1711-1722$

22. Tesch GH (2010) Macrophages and diabetic nephropathy. Semin Nephrol 30:290-301

23. Lim AK, Ma FY, Nikolic-Paterson DJ, Kitching AR, Thomas MC, Tesch GH (2010) Lymphocytes promote albuminuria, but not renal dysfunction or histological damage in a mouse model of diabetic renal injury. Diabetologia 53:1772-1782

24. Zheng F, Cornacchia F, Schulman I et al (2004) Development of albuminuria and glomerular lesions in normoglycemic B6 recipients of $\mathrm{db} / \mathrm{db}$ mice bone marrow: the role of mesangial cell progenitors. Diabetes 53:2420-2427

25. Kokkola R, Andersson A, Mullins G et al (2005) RAGE is the major receptor for the proinflammatory activity of HMGB1 in rodent macrophages. Scand J Immunol 61:1-9

26. Lewis EJ, Greene T, Spitalewiz S et al (2012) Pyridorin in type 2 diabetic nephropathy. J Am Soc Nephrol 23:131-136

27. Lim AK, Ma FY, Nikolic-Paterson DJ, Thomas MC, Hurst LA, Tesch GH (2009) Antibody blockade of c-fms suppresses the progression of inflammation and injury in early diabetic nephropathy in obese $\mathrm{db} / \mathrm{db}$ mice. Diabetologia 52:1669-1679 